\title{
A CARACTERIZAÇÃO DO DESIGN THINKING COMO UM MODELO DE INOVAÇÃO
}

\author{
Mayara Atherino Macedo \\ Mestrado em Engenharia de Produção pela Universidade Federal de Santa Catarina - UFSC \\ Professor da Faculdade do Vale Itajaí Mirim - FAVIM \\ m.atherinomacedo@gmail.com (Brasil)
}

\section{Paulo Augusto Cauchick Miguel}

Doutorado em Manufacturing Engineering pela University of Birmingham, Inglaterra

Professor da Universidade Federal de Santa Catarina - UFSC

cauchick@ufsc.br (Brasil)

\section{Nelson Casarotto Filho}

Doutorado em Engenharia de Produção pela Universidade Federal de Santa Catarina - UFSC nelson.casarotto@ufsc.br (Brasil)

\section{RESUMO}

Para as organizações manterem-se competitivas em um mercado que está em constante mudança, é preciso ofertar soluções inovadoras, atendendo às necessidades do consumidores. Para gerar inovações alinhadas às estratégias empresariais são necessários processos de coordenação das atividades dos diferentes setores da empresa, em outras palavras, é preciso que seja desenvolvido um processo de inovação na organização. Na última década o design thinking vem sendo utilizado para promover a inovação nas organizações, neste contexto, surge o questionamento se a área realmente contribui para gerar inovação e se abrange toda a gama de tipos de inovação. Com isso, o objetivo do trabalho é caracterizar o design thinking como um modelo de inovação. A pesquisa tem caráter teórico conceitual, através do desenvolvimento de uma revisão da literatura sobre inovação, e análise de publicações com dados empíricos sobre design thinking. A análise dos dados empíricos evidenciou que o design thinking é indicado para inovações abertas, sendo possível gerar inovações de várias naturezas (radical, semirradical e incremental) e tipos (produto, serviço, processo, organizacional e de marketing). A pesquisa concluiu, com base nos requisitos essenciais de um processo de inovação e nas principais características de um modelo de inovação, que o design thinking pode ser caracterizado como um modelo de inovação.

Palavras-chave: Inovação; Processos de inovação; Modelos de inovação; Design thinking. 


\section{INTRODUÇÃO}

Schumpeter (1984) afirma que a inovação é o motor fundamental do crescimento econômico, assim a dinâmica atual do ambiente competitivo global estimula as empresas a buscar vantagens competitivas e diferenciação do mercado, para obter melhores resultados econômicos. Para Brown (2008), a inovação se tornou a principal fonte de diferenciação e vantagem competitiva para as organizações. Com isso, as organizações buscam modelos mais eficientes para aperfeiçoar os processos de inovação, criando novas maneiras de atender às demandas dos consumidores (Baregheh, Rowley \& Sambrook, 2009; Drucker, 2002; Liem \& Brangier, 2012).

Um processo de inovação engloba etapas que orientam as atividades de inovação desde a geração de ideias até a implementação da solução (Bonini \& Sbragia, 2011). Para possibilitarem aplicação prática, os processos de inovação são estruturados em modelos, que compreendem práticas, procedimentos e políticas para o desenvolvimento de inovações (Longanezi, Coutinho \& Bomtempo, 2008). A partir da década de 50 surgiu uma série de modelos de inovação, que utilizaram desde processos lineares e simples a uma estrutura de atividades mais complexa e voltada às demandas do mercado (Rothwell, 1994). Desenvolver inovações é imprescindível para uma empresa manter a competividade no mercado, assim, o uso de modelos para gerar inovação é essencial para as organizações alcançarem seus objetivos estratégicos.

Na última década o design thinking vem sendo utilizado como um meio de desenvolver inovações nas organizações (Brown, 2008). O design thinking envolve o processo de geração de ideias em um grupo multidisciplinar como foco na resolução de problemas, assim, a utilização do design vai além da aparência dos produtos, sendo aplicado na concepção de soluções, que englobam os aspectos estratégicos do negócio (Bonini \& Sbragia, 2011). Neste contexto, em que o design thinking é utilizado para promover a inovação, surge o questionamento se a área de conhecimento realmente contribui para gerar inovação, abrangendo toda a gama de tipos de inovação. Assim, o objetivo deste trabalho é caracterizar o design thinking como um modelo de inovação.

Esta a pesquisa é dividida em seis seções, incluindo esta introdução. A seção dois apresenta os conceitos e tipos de inovação, características dos processos de inovação e os modelos de inovação. A seção três apresenta as definições sobre design thinking. O item quatro descreve os métodos de pesquisa adotados, enquanto a seção cinco aborda a discussão e análise dos dados. A última parte traz as conclusões.

Revista de Administração e Inovação, São Paulo, v. 12, n.3 p. 157-182, jul./set. 2015. 


\section{CONCEITO DE INOVAÇÃO}

Schumpeter (1984), pioneiro nos estudos sobre inovação, diferencia invenção, o momento quando a ideia é gerada, de inovação, o momento em que a ideia é implementada no mercado. Conforme o autor, a inovação é composta pela combinação dos meios de produção, atividades organizacionais e de marketing, sendo que a invenção contribui consideravelmente com a inovação.

A invenção pode ser compreendida como a concepção de algo inexistente, com uma finalidade especifica, registrada em algum meio físico, tal como projetos, planos, protótipos e etc.; enquanto a inovação é descrita como a introdução no mercado de algo novo, observando os aspetos técnicos, mercadológicos, econômicos e organizacionais (Barbieri, 2004). Mlecnik (2012) complementa a definição de inovação, afirmando ser todo o conhecimento novo desenvolvido para o lançamento de um produto ou serviço no mercado.

O Manual de Oslo, desenvolvido pela Organização para Cooperação e Desenvolvimento Econômico (OECD, 2004, p. 55) define a inovação como "a implementação de um produto (bem ou serviço) novo ou significativamente melhorado, ou um processo, ou um novo método de marketing, ou um novo método organizacional nas práticas de negócios, na organização do local de trabalho ou nas relações externas". A OECD (2004) considera inovação quando um produto, processo, método de marketing ou organizacional novo (ou significativamente novo) é inserido no mercado. A inovação é um processo multi-estágio pelo qual as empresas transformam ideias em produtos, serviços ou processos novos ou melhorados, visando a diferenciação no mercado e aumento da competitividade, alcançando o sucesso (Baregheh, Rowley \& Sambrook, 2009).

Sawhney; Wolcott e Arroniz (2011) afirmam que a inovação só é relevante quando cria valor para os clientes, um novo e substancial valor através de produtos e serviços; e para a empresa, por meio de mudanças em uma ou mais dimensões do sistema de negócios, utilizando a criatividade.

A inovação, na visão de Pärttö e Saariluoma (2012), tem a missão de resolver problemas, através de atividades de ideação, design, marketing de produto e vendas, além da difusão e aceitação dos usuários. Esta abordagem é a adotada neste artigo, combinada à definição de inovação do Manual de Oslo (OECD, 2004), o que possibilita identificar demandas para inovar em diversas áreas de uma empresa.

Revista de Administração e Inovação, São Paulo, v. 12, n.3 p. 157-182, jul./set. 2015. 


\subsection{Tipos de inovação}

A inovação pode ser classificada segundo o objeto, o grau de novidade e a origem da fonte. A inovação conforme o objeto é caracterizada no Manual de Oslo (OCDE, 2004) em quatro tipos: i) de produto: bens ou serviços novos ou substancialmente melhorados; ii) de processo: um novo ou significativamente melhorado método de produção ou distribuição; iii) de marketing: método de marketing novo (ou com alterações significativas) no conceito de um produto, na embalagem, em relação ao posicionamento no mercado, na promoção ou definição de preços; e iv) organizacional: novo método organizacional na gestão de empresa, na organização do local de trabalho ou interações externas, onde o foco é melhorar a performance organização.

Tidd; Bessant e Pavitt (2008) definem a inovação como um processo de mudança e apresentam o conceito dos 4 P's da inovação, na qual a classificam em inovação de produto, de processo (que inclui tanto alterações nos processos produtivos e administrativos da instituição), de posição (cujo conceito é similar à definição de inovação de marketing apresentada no Manual de Oslo), e de paradigma (mudanças no modelo de negócio da empresa). De modo geral, o tipo de inovação descrita por Tidd; Bessant e Pavitt (2008) e OECD (2004) apresentam caraterísticas muito similares, a principal diferença está na inovação de paradigma, que pode ser compreendido no Manual de Oslo como inovação organizacional.

A classificação da inovação conforme o grau de novidade consiste em: i) incremental: melhoria dos produtos e processos existentes (Davila, Epstein \& Shelton, 2008; Tidd, Bessant \& Pavitt, 2008); ii) semi-radical: envolve uma mudança significativa no modelo de negócio ou na tecnologia utilizada pela empresa, que não seriam alcançadas com a inovação incremental (Davila, Epstein \& Shelton, 2008); e iii) radical: oferta de produtos e processos de maneira totalmente nova (para o mundo ou para a empresa), causando alterações no setor industrial a qual pertencem (Davila, Epstein \& Shelton, 2008; Tidd, Bessant \& Pavitt, 2008).

Um modelo de negócio descreve a maneira como uma empresa cria e entrega valor (ou benefícios) aos seus clientes, envolvendo os clientes, oferta (produtos/serviços), infraestrutura e viabilidade financeira (Osterwalder \& Pigneur, 2011). A tecnologia é um dos elementos que influencia na concepção do modelo de negócio, uma vez que o seu uso poderá resultar em novos benefícios aos consumidores (Teece, 2010).

Assim, Davila, Epstein e Shelton (2008), afirmam que interação entre as características do modelo de negócio e os aspectos tecnológicos resultam em diferentes graus de inovação. Conforme os autores, o desenvolvimento de um portfolio de produtos e serviços que equilibra os diferentes graus de

Revista de Administração e Inovação, São Paulo, v. 12, n.3 p. 157-182, jul./set. 2015. 
novidade é essencial para gerar um crescimento sustentado. A figura 1 apresenta a matriz da inovação, onde a interação entre a tecnologia e o modelo de negócios da empresa indica o tipo de inovação implantada.

\section{Figura 1}

Matriz da inovação.

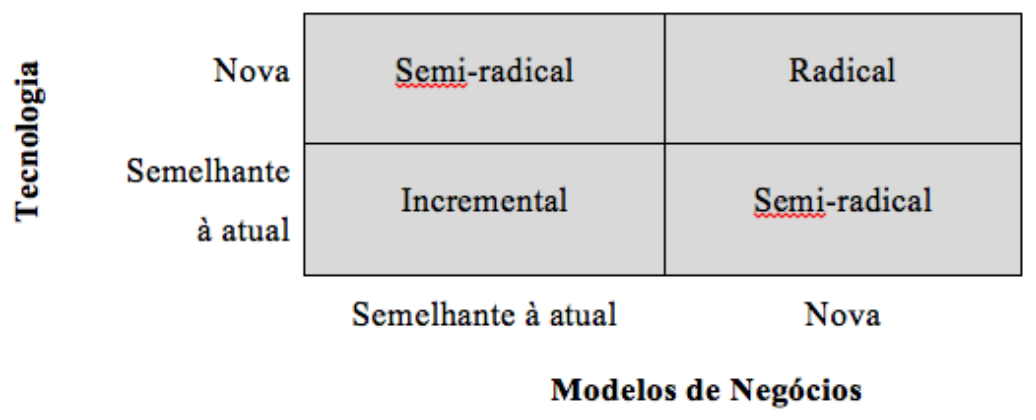

Fonte: Davila; Epstein e Shelton (2008, p. 34)

Chesbrough (2012) classifica a inovação com base na origem da fonte, podendo ser interna (inovação fechada) ou externa (inovação aberta). Para o autor a inovação fechada constitui um modelo de integração vertical tradicional onde a organização possui maior controle sobre o processo, cuja demanda para inovação advém de fontes internas (P\&D, produção ou outras áreas funcionais da organização), e os produtos e serviços são desenvolvidos internamente e, então, ofertados ao mercado. Conforme o autor, projetos com inovação fechada são desenvolvidos com base cientifica e tecnológica da empresa e apresentam apenas uma entrada (o P\&D) e uma saída (o mercado).

$\mathrm{Na}$ inovação aberta, conforme Chesbrough (2012), o processo é mais colaborativo e envolve uma série de participantes, podendo ser consumidores, instituições de ensino, fornecedores, parceiros e comunidade em geral. A inovação aberta é gerada a partir da união de competências internas e ideias externas, oferecendo ao mercado algo novo e condizente às suas necessidades (Rodrigues, Heringer \& França, 2010). De acordo com Chesbrough (2012), este tipo de inovação é gerada a partir de pesquisas de prospecção e análise das oportunidades, tendo várias entradas (clientes, fornecedores, etc.) e uma saída (o mercado).

Assim, a inovação pode se entendida como a introdução no mercado de produtos, processos, ações de marketing ou processos organizacionais diferentes dos atuais ou melhorados. Pode ser qualificada com base no objeto de inovação (produto, processo, marketing e organizacional), no nível de inovação (radical, semi-radical e incremental) e onde é gerada (interna ou externamente). 


\subsection{Processo de inovação}

Um processo de inovação compreende um conjunto de atividades ordenadas e integradas para geração de valor para os consumidores, por meio produtos, serviços e informações (Cruz, 2003; Oliveira, 2008).

Wylant (2008) afirma que para alcançar a inovação é necessário colocar em prática algum tipo de processo que irá produzir algo novo. Para o autor, o processo de inovação nasce no momento em que se realiza uma ação, inspirada por uma ideia, resultando na inserção de um novo produto, sistema, processo ou modelo de negócio no mercado. Assim, qualquer atividade posterior à ideia, que visa a materializar um conceito, compreende o processo de inovação.

O processo de inovação, consiste em um caminho organizado para a resolução de problemas, partindo de operações sistemáticas e perseverantes, combinando conhecimento, habilidades e comportamento de um individuo (Pärttö \& Saariluoma, 2012; Verganti \& Öberg, 2013).

O processo de inovação, conforme Gordon, Tarafdar, Maksimoski e Rogowitz (2008), compreende as atividades e habilidades necessárias para gerar algo novo, desde a concepção até o lançamento no mercado. A compreensão da maneira como as fases do processo de inovar são desenvolvidas e a relação entre elas consiste em um elemento importante para entender como a inovação é gerada.

O processo de inovação é composto por um conjunto de atividades estruturadas e padronizadas que envolvem a identificação e análise de uma oportunidade, geração e seleção de ideias e conceito, até o lançamento no mercado (Hacklin, Inganas, Marxt \& Pluss 2009; Koen et al., 2001). Tohidi e Jabbari (2012) apresentam uma definição complementar ao processo de inovação, definindo-o em atividades estruturadas de operações técnicas industriais e comerciais, para gerar ideias de processos, produtos ou serviços novos. Assim, um processo de inovação consiste em atividades de produção e comercialização de novo produto/serviço ou implementação de um novo processo na empresa.

\subsection{As caraterísticas de um processo de inovação}

Para Schumpeter (1984), o processo de inovação é constituído, basicamente, por três fases: a invenção, a inovação e a difusão. Para o autor, a invenção compreende a descoberta da oportunidade comercial, a geração de ideias e os novos princípios técnicos; a inovação consiste na transformação da ideia em algo comercial; e a difusão caracteriza a expansão de uma inovação que já foi introduzida no mercado em novos produtos e processos. Cada uma destas fases apresentam caraterísticas específicas.

Revista de Administração e Inovação, São Paulo, v. 12, n.3 p. 157-182, jul./set. 2015. 
O processo de inovação empresarial é sistêmico, englobando todas as dimensões de um negócio, ou seja, inclui os processos desenvolvidos pela empresa para criar e entregar valor aos consumidores (Sawhney, Wolcott \& Arroniz, 2011). Os autores reuniram quatro principais dimensões de um sistema de negócios pelo qual uma empresa pode buscar oportunidades para gerar a inovação, são elas:

a) Oferta (o que): plataforma e soluções. Consiste na tecnologia empregada para produzir um bem que atende uma oportunidade de mercado.

b)Consumidores (quem): experiência do cliente e captura de valor. Incide na forma como o público recebe a oferta de empresa, se ele compreende a relevância do item e este atende às necessidades e desejos.

c) Presença (onde): networking e marca. É o contato do consumidor com o produto, através dos pontos de venda, canais de comunicação e a marca.

d)Processos (como): organização e a cadeia de suprimentos. São os processos internos desenvolvidos na organização que viabilizam a produção do produto.

A proposta de Sawhney, Wolcott e Arroniz (2011) apresenta alguns elementos que interferem no processo de inovação, envolvendo a origem do conhecimento e os ambientes onde se pode iniciar o processo de inovação. Porém, a proposta dos autores enfatiza a inovação do produto, e as dimensões apresentadas estão mais relacionados com as características da inovação segundo a fonte de origem, que pode ser externa ou interna. Sendo assim, a proposta de Sawhney, Wolcott e Arroniz (2011) ainda deixa lacunas quanto às características de um processo de inovação, visto que aborda apenas um objeto e um tipo de inovação.

Um estudo desenvolvido por Baregheh, Rowley e Sambrook (2009) identificou os seis atributos mais importantes do processo de inovação, que combinados com os conceitos de inovação de Davila, Epstein e Shelton (2008), Tidd, Bessant e Pavitt, (2008), Schumpeter (1984) e OECD (2004), podem ser resumidos em um diagrama que apresenta a essência da inovação (figura 2). O esquema não constitui um fluxo linear e sua importância está nos estágios, ou seja, na apresentação de um possível fluxo do processo de inovação, indicando os vários pontos de partida do processo (Baregheh, Rowley \& Sambrook, 2009). 


\section{Figura 2}

Diagrama de requisitos essenciais do processo de inovação.

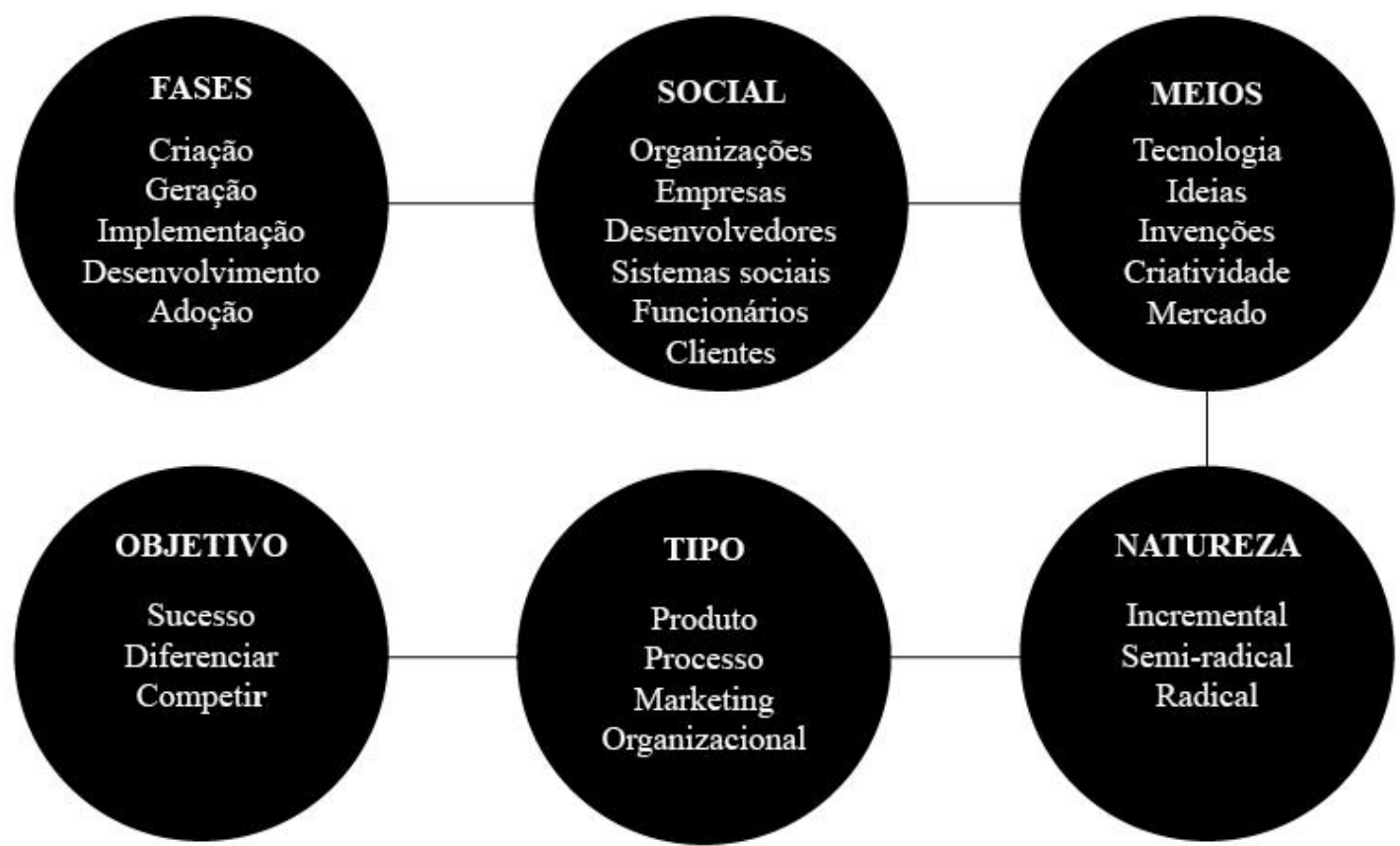

Fonte: Adaptado de Baregheh, Rowley e Sambrook (2009).

As fases da inovação compreendem os passos dados durante um processo de inovação; o contexto social compreende qualquer agente que interfere no processo de inovação (sejam pessoas, organizações ou fatores ambientais); o bloco dos meios de inovação estão relacionados aos recursos necessários para que a inovação ocorra; a natureza da inovação consiste no grau de novidade (radical, semi-radical e incremental); o tipo de inovação está relacionado ao resultado da inovação, ou seja, o objeto da inovação (produto, processo, marketing e organizacional); e o objetivo consiste no resultado que a empresa que alcançar ao desenvolver uma inovação, tendo relação com os objetivos estratégicos empresariais (Baregheh, Rowley \& Sambrook, 2009).

O diagrama dos requisitos essenciais do processo de inovação consiste em uma definição geral, mais genérica e abrangente, do processo de inovação, apresentando o processo como um esquema nãolinear que pode ser iniciado a partir de diversas fontes e áreas da organização (Baregheh, Rowley \& Sambrook, 2009). O processo oferece uma visão multidisciplinar que apoia e melhora a geração de inovações.

Para Hacklin et al. (2009) um fator chave para inovar com sucesso está na execução de um processo estruturado na condução de projetos, entretanto, o processo formalizado de inovação também pode ser uma limitação, dependendo das restrições e rigidez. Assim, os autores apresentam o processo 
de inovação stage-gate (figura 3), composto por uma serie de atividades prescritas, que possuem pontos de decisão. De acordo com os autores, tal processo apresenta as fases sequencialmente, porém, permite a realização simultânea de varias atividades dentro de cada fase.

\section{Figura 3}

Processo de inovação stage-gate.

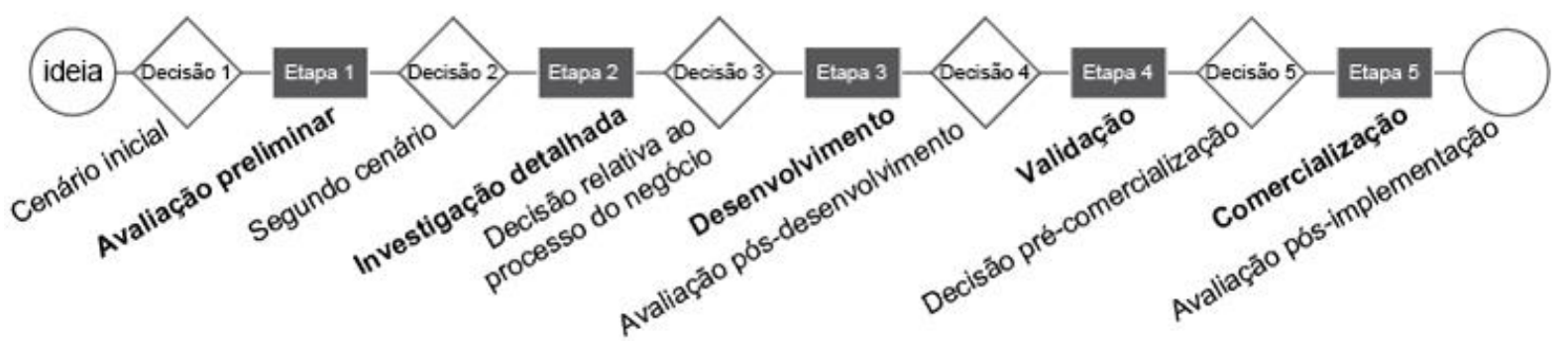

Fonte: Hacklin et al. (2009) adaptado de Cooper (1990, p. 46)

O processo apresentado por Hacklin et al. (2009) é um esquema genérico, porém traz um elemento novo, os pontos de análise do projeto em cada etapa e a decisão dos passos seguintes. Apesar desta análise pontual ser um item importante a inovação, o processo está mais focado nas etapas após o início do desenvolvimento da inovação, não evidenciando a fonte de origem da inovação, ao contrário da proposta de Baregheh, Rowley e Sambrook (2009) que exibe as possíveis entradas do processo de inovação.

Enquanto um processo de inovação apresenta os elementos da inovação (as fases), um modelo de inovação descreve as práticas, os procedimentos e as políticas que devem compor os processos de desenvolvimento de inovações (Longanezi, Coutinho \& Bomtempo, 2008; Oliveira, 2008). Assim, para uma organização gerar uma inovação é essencial seguir um fluxo de ações ordenadas, caraterizadas como um mapa da inovação, representados através de modelos. A seção a seguir apresenta seis modelos de inovação.

\subsection{Modelos de inovação}

Após a segunda guerra mundial houve o crescimento econômico das indústrias, o desenvolvimento do mercado e o aumento da competição, com isso, a inovação passou a ser vista como um propulsor para o incremento da produtividade e a manutenção da competitividade empresarial (Rothwell, 1994). Assim, conforme Saraiva e Rolo (2009), a partir de década de 50 surgiram teorias para explicar o fluxo do processo de inovação, dando origem a cinco gerações de 
modelos lineares de inovação e, mais tarde, um modelo interativo denominado chain-link ou modelo das ligações em cadeia.

O primeiro modelo de inovação (figura 4), conhecido como inovação de primeira geração ou tecnologia push (1950 - 1960), prevê um fluxo unidirecional e concebe a inovação a partir da pesquisa básica, seguida pela pesquisa aplicada, o desenvolvimento no departamento de $\mathrm{P} \& \mathrm{D}$, a produção e a difusão da inovação, não ocorrendo a realimentação entre a fases (Edquist \& Hommen, 1999; Godin, 2006; Hobday, Boddington \& Grantham, 2012a; Wang \& Kleiner, 2005).

\section{Figura 4}

Modelo de inovação de primeira geração.

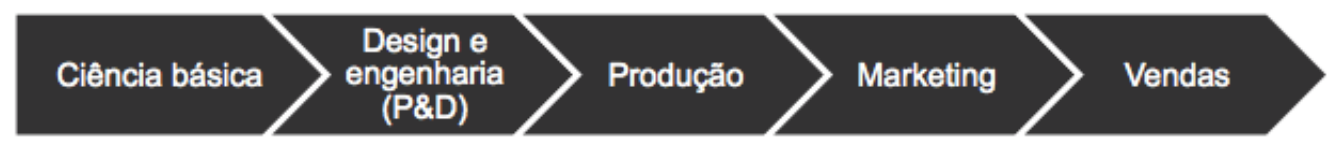

Fonte: Wang e Kleiner (2005, p. 88)

O modelo de inovação de primeira geração é simplista e de pouca aplicação prática para a grande maioria das indústrias, visto que as necessidades do consumidor são definidas pelo departamento de P\&D e não pelo mercado (Edquist \& Hommen, 1999; Tohidi \& Jabbari, 2012).

O modelo de inovação da segunda geração ou demand pull (1960 - 1970) (figura 5), também foi um processo linear simples, porém, devido a crescente ênfase estratégica em marketing, as organizações passaram a buscar no mercado as ideias para o desenvolvimento de produtos (Hobday, Boddington \& Grantham, 2012b; Rothwell, 1994; Wang \& Kleiner, 2005). Neste modelo, o mercado direciona o P\&D da empresa, ou seja, os consumidores são fontes de ideias para o desenvolvimento de novos produtos, e a função do P\&D passa ser o atendimento das demandas do mercado

Os dois primeiros modelos de inovação não apresentam uma etapa de validação das necessidades e/ou do produto após o lançamento no mercado, como também não possuem a retroalimentação entre as fases. Hronszky e Kovács (2013) afirmam que os modelos de inovação lineares consistem em inovações fechadas, visto que a demanda é gerada pelo departamento de P\&D da empresa. Conforme os autores, só é possível alcançar sucesso com a inovação linear fechada quando há uma série de fatores coincidentes colaborando, tais como uma demanda estável, clientes satisfeitos com produtos de massa e consumidores passivos - fatores praticamente impossíveis de alcançar nos dias atuais - evidenciando a deficiência e pouca aplicação prática destes modelos. 


\section{Figura 5}

Modelo de inovação de segunda geração.

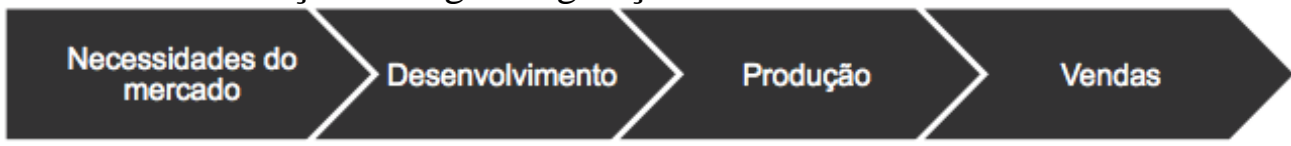

Fonte: Rothwell (1994, p. 9)

O modelo de inovação de terceira geração (1970 - 1980) (figura 6), é um processo mais estruturado, através da combinação dos dois modelos anteriores, equilibrando as capacidades tecnológicas com as demandas do mercado (Hobday, Boddington \& Grantham, 2012b; Rothwell, 1994; Wang \& Kleiner, 2005). Foi o primeiro modelo que não linear, sendo sequencial e dividido em fases distintas, mas que interagem entre si, onde o foco está no feedback entre o P\&D e o marketing.

\section{Figura 6}

Modelo de inovação de terceira geração.

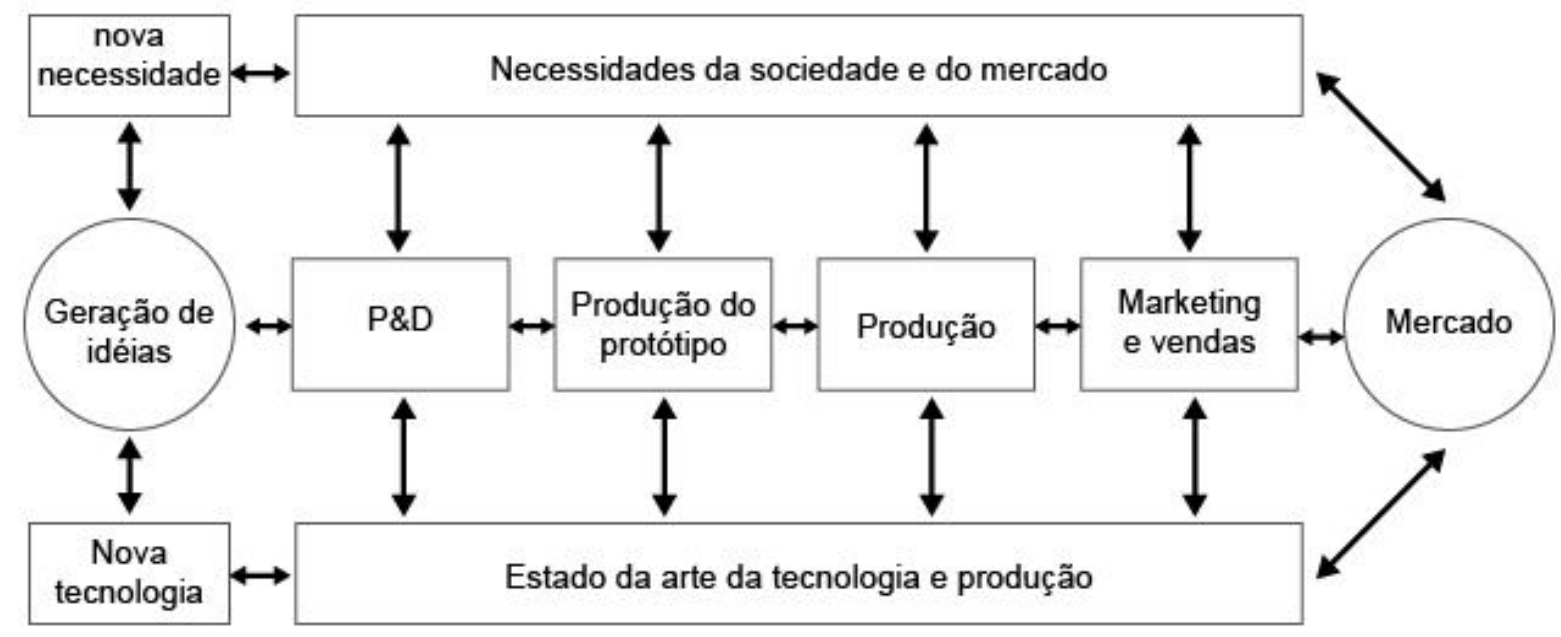

Fonte: Rothwell (1994, p. 10)

O modelo de inovação de quarta geração (1980 - 1990), tem como principal característica a integração e o desenvolvimento paralelo das atividades de criação de um produto. Além dos fortes vínculos com os fornecedores, integrando-os ao estágio inicial de desenvolvimento de produtos, os diferentes departamentos da empresa trabalham simultaneamente no projeto (Hobday, Boddington \& Grantham, 2012b; Rothwell, 1994; Wang \& Kleiner, 2005). O modelo apresenta grandes avanços em relação aos anteriores, além do feedback do mercado, há também a interação entre os atores do processo e as etapas de desenvolvimento.

O modelo de inovação de quinta geração ou modelo de rede (após 1990), enfatiza o aprendizado que surge dentro de organização e entre empresas, sugerindo um processo de rede distribuída (İzadi, Zarrabi \& Zarrabi, 2013). Neste modelo, há maior integração entre os atores da 
cadeia de produção (clientes, distribuidores, fornecedores, etc.), e o uso de recursos avançados de tecnologia da informação, visando a aumentar a velocidade e eficiência no desenvolvimento de novos produtos (Hobday, Boddington \& Grantham, 2012b).

O sexto modelo de inovação, o chain-link (figura 7), enfatiza a interação constante entre as oportunidades de mercado, a base de conhecimentos e competências da empresa (Alvares, 2010). No chain-link o desenvolvimento técnico se a partir de uma demanda do mercado, cujo projeto é desenvolvido e prototipado, dando início a um ciclo de retroalimentação em relação às necessidades do consumidor (Caraça, Lundvall \& Mendonça, 2009). Ao longo do desenvolvimento do produto, são utilizados conhecimentos pré-existentes na organização, mas novas experiências podem ser desenvolvidas internamente ou adquiridas externamente, a partir da interação com a rede de relacionamentos da empresa (Alvares, 2010; Caraça, Lundvall \& Mendonça, 2009).

\section{Figura 7}

Modelo de inovação chain-link.

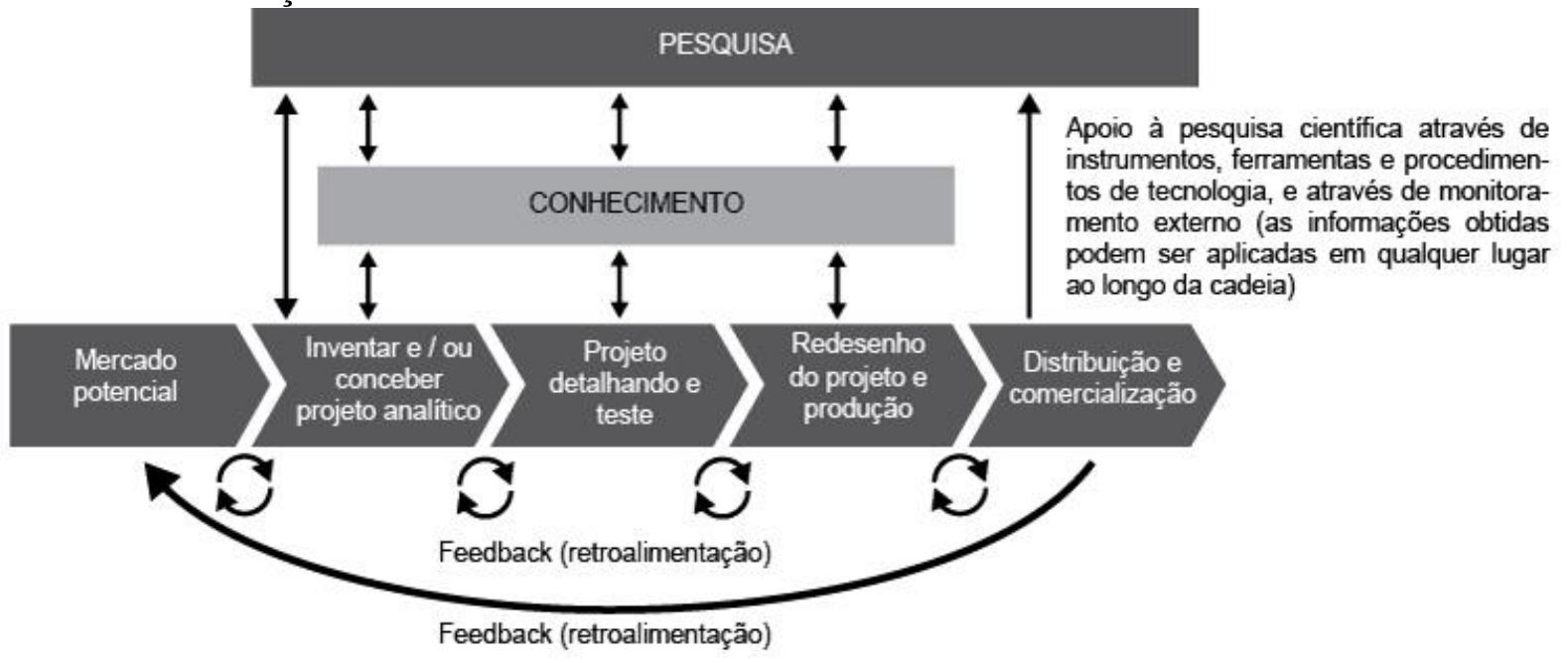

Fonte: Kline e Rosenberg (1986, p. 290)

O chain-link consiste em um modelo de inovação não linear, cujo ponto principal está na interação entre as demandas do mercado, os conhecimentos, habilidades e competências da empresa. Neste modelo, a inovação parte da identificação de uma oportunidade de mercado, que será desenvolvida a partir de um projeto, onde a pesquisa contribui para o processo de criação da inovação.

Dentre os modelos de inovação apresentados, percebe-se que houve mudanças ao longo do tempo, porém, de acordo com Hobday; Boddington e Grantham (2012b), até o modelo de terceira geração o foco estava nos processos de P\&D. A partir dos anos 70, começaram a surgir modelos mais complexos que enfatizavam a interação entre as fases do desenvolvimento do produto e o mercado (Tohidi \& Jabbari, 2012). Desde o segundo modelo de inovação, as necessidades do mercado 
começam a ganhar importância, e a partir da terceira geração, há uma maior preocupação com o a interação entre os agentes do processo de inovação. O modelo de quinta geração e o chain-link acrescentam a troca de conhecimento e o aprendizado como fatores que contribuem para o processo de inovação.

Assim, pode-se entender que os elementos mais importantes de um modelo de inovação são o atendimento das demandas do mercado, a interação entre os agentes do processo de inovação, o aprendizado e o diálogo entre todos estes fatores.

\subsection{Design thinking}

Conforme Bonini e Sbragia (2011) o design thinking consiste em uma abordagem colaborativa de resolução de problemas, centrada no usuário, que gera inovação através de iteração e práticas criativas. Corroborando com esta visão, Bukowitz (2013) afirma que o design thinking consiste em uma abordagem de solução de problemas, que utiliza ferramentas de criatividade e conceitos de diversas disciplinas para encontrar uma solução.

O design thinking, conforme Brown (2008), pode ser entendido como um método que permeia as atividades de inovação com foco no ser humano, através de um processo de compreensão detalhado (por meio de observações diretas), para extrair os desejos e necessidades das pessoas não descritos em pesquisas tradicionais, tais como a entrevista.

O design thinking é um processo contínuo de reformulação da empresa a partir do ponto de vista do cliente, ou seja, o processo de geração de ideias envolve agentes externos à empresa (usuário, fornecedores, parceiros e etc.), para estimular, aprimorar e refinar as ideias (Liedtka, 2011; Fraser, 2012).

Cardon e Leonard (2010) por sua vez, asseguram que o design thinking alcança soluções inovadoras por meio da compreensão do contexto em que um projeto de inovação é desenvolvido. Os autores definem o termo como uma ferramenta que visa a compreensão, visualização e descrição de problemas complexos para, através da criatividade, auxiliar no desenvolvimento de soluções para tais problemas. Bukowitz (2013) apresenta uma visão similar à dos autores, alegando que o estudo do contexto contribui para compreender o verdadeiro problema, que pode ser redefinido, para que sejam geradas ideias de soluções inovadoras condizentes com a realidade do mercado.

Ao desenvolver uma inovação, é preciso que ela seja tecnologicamente viável, condizente com a estratégia de negócios da empresa, convertida em valor para o cliente e em oportunidade de mercado para a empresa (Brown, 2008). Assim, o autor apresenta as três fases que compõem o design thinking 
(figura 8): i) inspiração: o processo de descoberta centrado no ser humano; ii) ideação: atividades de geração, desenvolvimento e teste de ideias; e iii) implementação: mapeamento de um caminho para se chegar ao mercado.

Bonini e Sbragia (2011) afirmam que durante as três fases do design thinking o problema para o qual se busca uma solução é questionado, ideias são geradas e soluções são propostas. As etapas do design thinking não são lineares, constituindo ciclos iterativos, podendo ocorrer simultaneamente ou se repetirem, até se chegar a soluções inovadoras (Bonini \& Sbragia, 2011; Brown, 2008).

$\mathrm{O}$ processo de design thinking começa com a formação de equipes multidisciplinares que iniciam a etapa de inspiração com a identificação do problema que se deseja solucionar, bem como o público a quem se destina tal solução (Bonini \& Sbragia, 2011). Os autores sugerem a identificação das necessidades dos clientes, a tecnologia existente, e as competências e restrições da empresa. Também é importante observar e analisar o comportamento humano em determinadas situações para extrair novos conhecimentos (Brown, 2008). Nesta fase, o contexto de empresa e o consumidor são avaliados minuciosamente, para que nenhuma possível oportunidade seja deixada de lado.

A ideação parte dos problemas levantados na fase anterior para gerar ideias e conceitos, que serão convertidos rapidamente em protótipos, cuja função é avaliar os pontos fortes e fracos da proposta, de acordo com Bonini e Sbragia (2011). A prototipagem rápida faz parte do processo de concepção da ideia, visto que transforma uma ideia em algo tangível e, às vezes, em modelos experimentais, permitindo visualizar o conceito e criar novas soluções (Brown, 2008; Liedtka, 2011). 


\section{Figura 8}

Etapas do processo de design thinking.

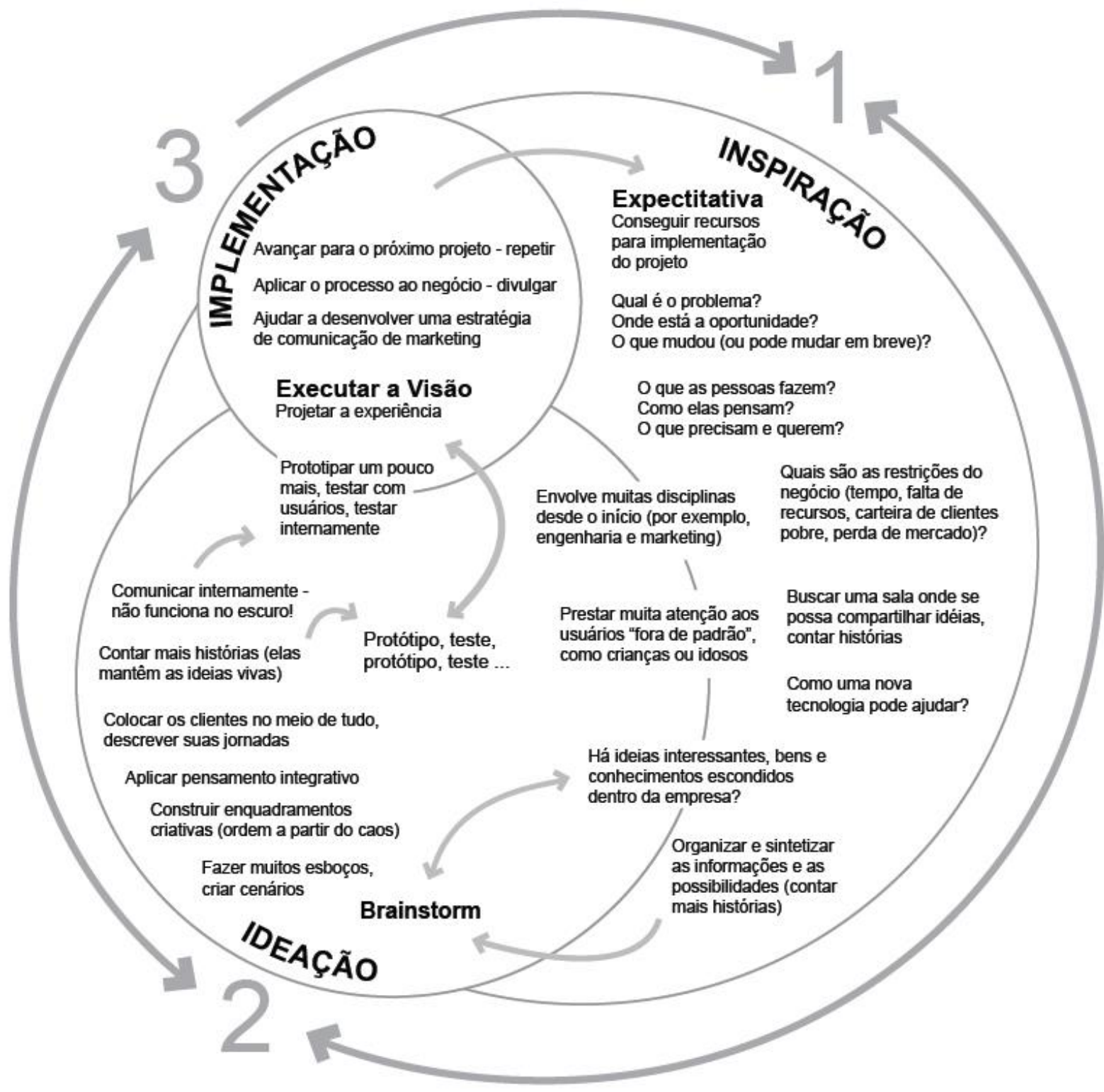

Fonte: Brown (2008, p. 6)

$\mathrm{Na}$ fase da implementação a solução é definida, planejada e produzida com base nos preceitos listados por Brown (2008), de tecnologia praticável, viabilidade para o negócio e geração de valor para o consumidor. Conforme o autor, a organização deve, neste momento, definir as ações estratégias, operacionais e econômicas para viabilizar a inserção da solução no mercado.

Então, o design thinking pode ser compreendido com um meio de solução de problemas, composto por três fases não lineares - inspiração, ideação e implementação -, que podem ocorrer em simultaneidade (Brown, 2008, Bonini \& Sbragia, 2011). O processo ocorre por meio da compreensão 
do contexto do problema (com foco nas atividades do ser humano) e na ressignificação do problema descrito. As três fases do design thinking podem ser desdobradas em atividades, onde a etapa de inspiração engloba a pesquisa de mercado; a ideação envolve a geração e teste de ideias, desenvolvimento e teste de protótipos; e a implementação consiste na produção e execução da solução encontrada na etapa anterior, com base na tecnologia disponível, competências da organização e demandas do consumidor (Brown, 2008; Liedtka, 2011; Bonini \& Sbragia, 2011).

Entretanto, conforme afirma Liedtka (2011) o design thinking é um processo de co-criação, envolvendo clientes em potencial para contribuir no processo de desenvolvimento de novas ofertas de negócios, seja por meio da descrição de insatisfações, sugestão de ideias ou teste de protótipos. Assim, é possível afirmar que os fatores que diferenciam o design thinking dos demais modelos de inovação consistem no estudo detalhado do contexto do problema (com possibilidade de ressignificação), e a cocriação que ocorre entre a empresa, os clientes, fornecedores e demais pessoas envolvidas no processo de desenvolvimento e/ou uso do produto/serviço.

\section{MÉTODOS E TÉCNICAS DE PESQUISA}

Este trabalho se caracteriza como teórico conceitual, uma vez que se apoia na revisão de literatura para gerar um conhecimento inicial sobre inovação e design thinking, que auxiliou o processo de formulação e clarificação do tema da pesquisa, contribuiu para a etapa de análise de dados e na descrição da discussão e resultados (Miguel et al., 2009; Seuring \& Gold, 2012).

Partindo do problema de pesquisa “o design thinking é um processo de inovação?”, desenvolveu-se a busca nas bases de dados Scielo, Science Direct, Scopus e Web of Science objetivando obter material para construir o referencial teórico. A coleta de dados partiu de um levantamento de artigos científicos de periódicos (com delimitação temporal de 5 anos) e livros. Buscou-se obter publicações de caráter descritivo sobre inovação e artigos sobre design thinking com dados empíricos, que explicassem o uso prático do design thinking. O quadro 1 apresenta um fluxo com as etapas para a execução do trabalho.

Assim, a pesquisa foi conduzida a partir da busca e seleção de estudos para uma avaliação preliminar da literatura, visando formular e esclarecer o tema de pesquisa, identificar as oportunidades de pesquisa, definir a problemática e objetivo do estudo. Em seguida foi desenvolvida a avaliação crítica das publicações, através da análise dos objetivos, construtos e principais resultados dos artigos;

Revista de Administração e Inovação, São Paulo, v. 12, n.3 p. 157-182, jul./set. 2015. 
após a síntese dos dados, foram desenvolvidos os resultados, discussão e a concepção do relatório (Galvão, Sawada \& Trevizan, 2004; Seuring \& Gold, 2012).

\section{Quadro 1}

Fluxo das etapas macro para a execução do trabalho.

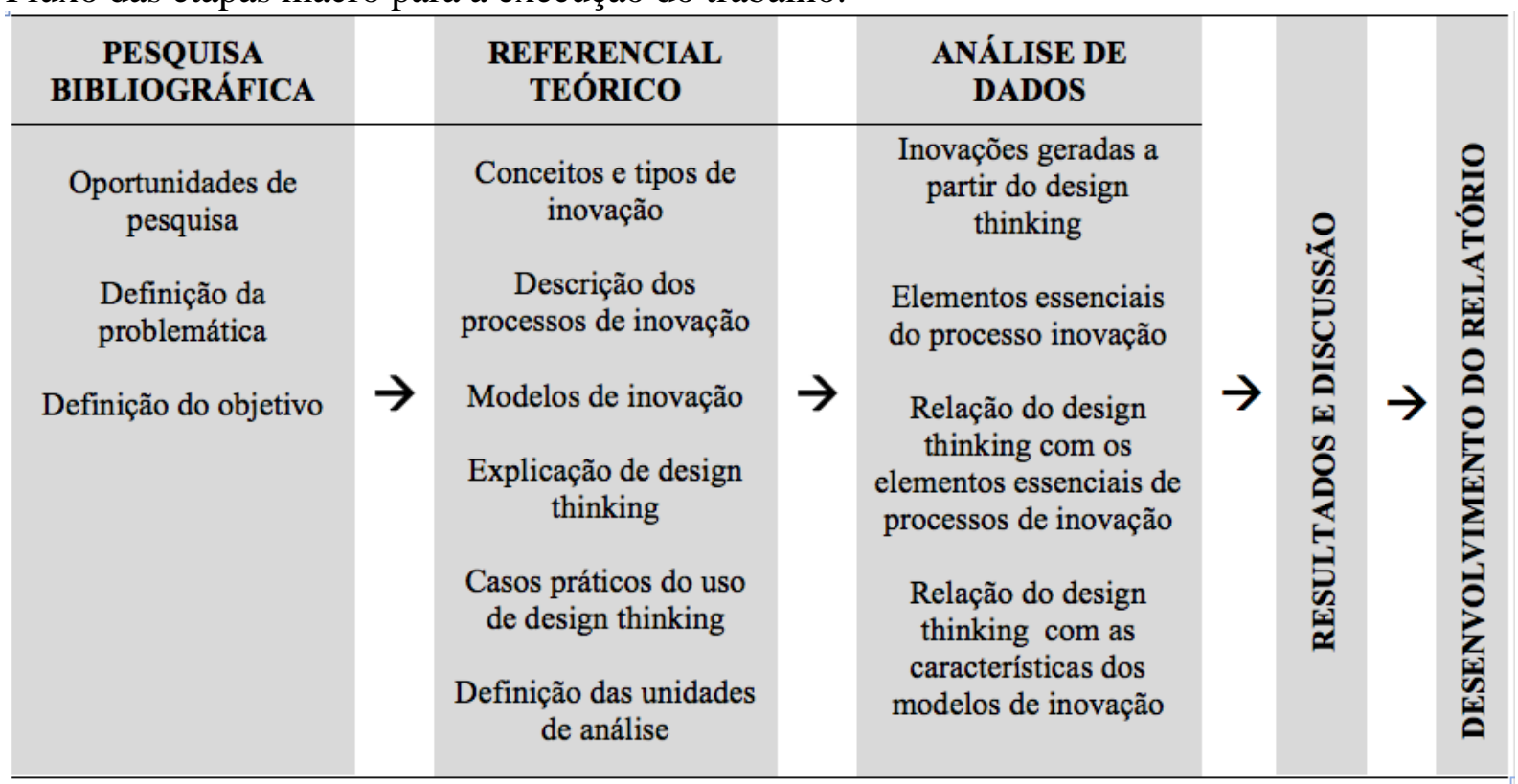

Fonte: baseado em Seuring e Gold (2012) e Galvão, Sawada e Trevizan (2004).

O arcabouço teórico-conceitual apresentou os conceitos e tipos de inovação, forneceu a compreensão dos processos e modelos de inovação, além das definições de design thinking, com suas respectivas fases, expondo situações reais de aplicação do design thinking em empresas. Após a análise das publicações com dados empíricos de inovações através do design thinking, foram selecionados os casos com base no tipo de inovação gerada (produto, marketing, processo e organizacional). O referencial teórico forneceu as informações sobre os elementos essenciais de um processo de inovação e as principais características de um modelo de inovação.

A análise de conteúdo dos artigos sobre design thinking ocorreu com base nas unidades de análise - tipos de inovação, elementos essenciais de um processo de inovação, características de um modelo de inovação - definidas a partir do referencial teórico e sob o ponto de vista dos elementos do processo de inovação, a partir do diagrama adaptado de Baregheh, Rowley e Sambrook (2009) (figura 2). O uso do diagrama se mostrou eficaz, pois se trata de um desenho genérico e abrangente do processo de inovação, permitindo que seja aplicado em diferentes contextos, uma vez que agrega as etapas de desenvolvimento de uma solução com as diversas classificações da inovação. A análise dos dados permitiu a discussão sobre a possibilidade do design thinking gerar inovação, e se a área apresenta os elementos essenciais de um processo e de um modelo de inovação. 


\subsection{Inovação através do design thinking}

Brown (2008) e Liedtka (2011) apresentam cinco casos de empresas que desenvolveram inovações através do design thinking: a organização da saúde americana Kaiser Permanente; a multinacional fabricante de peças para ciclismo Shimano; a instituição indiana Aravind Eye Care System, maior prestador mundial de cuidados oftalmológicos de alta qualidade a baixo custo; a organização americana de serviços financeiros Bank of America; e a empresa de produtos para saúde e higiene Pfizer Consumer Products.

Brown (2008) descreve que foram definidas equipes de trabalho formadas por funcionários das empresas e consultores externos para entender o consumidor. Conforme o autor, a etapa de imersão permitiu às equipes obter um conhecimento mais detalhado da vida do consumidor, gerando um novo ponto de vista do problema inicial, contribuindo para a geração de novas ideias na etapa de ideação. Conforme apresenta Brown (2008), as fases seguintes consistiram em ciclos iterativos de criação de protótipos, testes e refinamento para implementação da solução. Para o autor, a solução do problema surgiu da observação do comportamento do consumidor, e a inovação ocorreu como uma consequência do design thinking.

Conforme relata Brown (2008), no caso da Kaiser Permanente, identificou-se que os pacientes estavam descontentes com o processo da troca de turno da equipe de enfermagem. O processo de design thinking gerou a reorganização do processo de mudança de turnos, com as informações sendo repassadas à nova equipe na frente do paciente, além de se iniciar o uso de um software para acrescentar dados ao longo do período de trabalho e recuperar as anotações dos funcionários de outros plantões. A mudança resultou na redução de mais de 50\% do tempo médio na mudança dos turnos, além da maior qualidade na transmissão de informações sobre os enfermos. O impacto principal da inovação na organização foi o aumento da produtividade.

Brown (2008) também apresenta o caso da Shimano, que desenvolveu um novo conceito de bicicleta, ao invés de uma nova tecnologia. Criou-se conceito de bicicletas Coasting, com foco no lazer, evidenciando a atividade de pedalar como algo simples e divertido. Em parceria com três grandes fabricantes, a Shimano desenvolveu bicicletas com estética e características técnicas similares às das bicicletas utilizadas antigamente (freio contra pedal, marcha automática e bancos mais confortáveis). Além disso, a empresa desenvolveu estratégias de varejo com foco no público-alvo e campanhas de relações públicas identificando os locais seguros para pedalar. Depois do lançamento em 2007, mais sete marcas procuraram a Shimano para fabricar bicicletas Coasting.

Revista de Administração e Inovação, São Paulo, v. 12, n.3 p. 157-182, jul./set. 2015. 
A Aravind Eye Care System, conforme descreve Brown (2008), pensou de forma sistêmica para oferecer cuidados oftalmológicos para a população rural pobre da Índia. O país possui 12 milhões de pessoas cegas em função da catarata, o que pode ser revertido através de cirurgia para substituição do cristalino opaco por lentes intraoculares. Porém, um dos maiores empecilhos que realização das cirurgias era o custo das lentes intraoculares, US\$200 o par. Para solucionar este problema, a empresa desenvolveu tecnologia para produzir as lentes ao custo de US\$ 4 cada. Atualmente a empresa fabrica 1 milhão de lentes, vendendo as excedentes por US\$5 para instituições de caridade e ONGs (Monteiro, 2013).

O Bank of America, conforme apresenta Brown (2008), desenvolveu um novo serviço baseado no comportamento do consumidor que, para poupar dinheiro, guardava moedas de troco dentro de potes em casa para, quando cheio, ser depositado na conta poupança. Assim, para integrar o comportamento do público-alvo criou-se nova conta poupança, a Keep the Change (fique com o troco), associada a um cartão de débito. Quando os clientes utilizam os cartões para fazer compras, podem optar por ter o total arredondado para cima e a diferença depositada em suas contas poupança. Em menos de 1 ano a empresa conquistou 2,5 milhões de novos clientes, 700 mil novas contas correntes e 1 milhão de novas contas poupança.

A Pfizer Consumer Products, segundo Liedtka (2011), montou uma equipe multifuncional para observar os clientes em casa, em todos os aspectos de suas vidas, para descobrir o que eles realmente queriam e precisavam. A pesquisa concluiu que muitos produtos para cuidados com a saúde são concebidos para utilização em casa, dificultando o transporte e uso em outros lugares. Assim, a Pfizer encontrou um caminho para inovar: desenvolveu produtos para cuidado com a saúde para ser utilizado fora de casa, os chamados produtos de bolso. A empresa criou uma série de produtos, e após testes piloto em algumas farmácias para avaliar a aceitabilidade, lançou no mercado toda uma linha de produtos de higiene e saúde de bolso. Tais produtos tornaram-se uma categoria que rende U\$ 500 milhões para a Pfizer.

A partir dos exemplos expostos anteriormente, desenvolveu-se a análise a partir da proposta do diagrama de requisitos essenciais do processo de inovação (figura 2), para verificar se design thinking apresenta tais caraterísticas (quadro 2). 


\section{Quadro 2}

Análise do design thinking sob o ponto de vista dos elementos essenciais do processo de inovação.

\begin{tabular}{|l|c|c|c|c|c|c|}
\hline \multicolumn{1}{|c|}{ Exemplo } & Objetivo \\
Permaner & $\begin{array}{c}\text { Atrair mais } \\
\text { pacientes e } \\
\text { reduzir } \\
\text { despesas }\end{array}$ & $\begin{array}{c}\text { Apresenta } \\
\text { todas }\end{array}$ & $\begin{array}{c}\text { Social } \\
\text { Inovação } \\
\text { aberta }\end{array}$ & Ideias & Incremental & Organizacional \\
\hline Shimano & $\begin{array}{c}\text { Impulsionar } \\
\text { as vendas de } \\
\text { bicicletas } \\
\text { para o } \\
\text { público } \\
\text { acima de } 40 \\
\text { anos de idade }\end{array}$ & $\begin{array}{c}\text { Apresenta } \\
\text { todas }\end{array}$ & $\begin{array}{c}\text { Inovação } \\
\text { aberta }\end{array}$ & Criatividade & $\begin{array}{c}\text { Semi- } \\
\text { radical }\end{array}$ & Marketing \\
\hline $\begin{array}{l}\text { Aravind Eye } \\
\text { Care System }\end{array}$ & $\begin{array}{c}\text { Erradicar a } \\
\text { cegueira } \\
\text { tratável na } \\
\text { Índia }\end{array}$ & $\begin{array}{c}\text { Apresenta } \\
\text { todas }\end{array}$ & $\begin{array}{c}\text { Inovação } \\
\text { aberta }\end{array}$ & Tecnologia & Radical & Processo \\
\hline Bank of America & $\begin{array}{c}\text { Atrair mais } \\
\text { clientes }\end{array}$ & $\begin{array}{c}\text { Apresenta } \\
\text { todas }\end{array}$ & $\begin{array}{c}\text { Inovação } \\
\text { aberta }\end{array}$ & Mercado & $\begin{array}{c}\text { Semi- } \\
\text { radical }\end{array}$ & Serviço \\
\hline $\begin{array}{l}\text { Pesenvolver } \\
\text { Products }\end{array}$ & $\begin{array}{c}\text { Um produto } \\
\text { inovador de } \\
\text { cuidados à } \\
\text { saúde }\end{array}$ & $\begin{array}{c}\text { Apresenta } \\
\text { todas }\end{array}$ & $\begin{array}{c}\text { Inovação } \\
\text { aberta }\end{array}$ & Mercado & $\begin{array}{c}\text { Semi- } \\
\text { radical }\end{array}$ & Produto \\
\hline
\end{tabular}

Fonte: Adaptado de Brown (2008) e Liedtka (2011).

Com base nos resultados dos casos apresentados por Brown (2008) e Liedtka (2011), pode-se compreender que as cinco organizações obtiveram êxito com a implantação da solução desenvolvida por meio do design thinking, alcançando o objetivo inicial.

O design thinking é compreendido por Brown (2008) como um método que permeia as atividades de inovação com foco no ser humano, cujas três fases - inspiração, ideação e implementação-, sob o ponto de vista do diagrama de requisitos essenciais do processo de inovação (figura 4), engloba todos os itens do bloco fase.

O contexto social está presente no design thinking, principalmente na fase de ideação, onde equipes compostas tanto por funcionários, como pelos clientes, fornecedores e demais atores necessários ao processo, são criadas para gerar ideias, intercambiando conhecimentos. O design thinking é um processo de co-criação, envolvendo equipes multidisciplinares (que incluem agentes externos às empresa), caracterizando o processo de inovação aberta, uma vez que se trata de um processo mais colaborativo e que envolve uma série de participantes e conhecimentos, internos e externos ( Liedtka, 2011; Chesbrough, 2012).

Os bloco dos meios de inovação pode ser visualizado no discurso de Brown (2008), ao afirmar que o design thinking deve propor soluções tecnologicamente viáveis, condizente com a estratégia de 
negócios da empresa, que gere valor para o cliente e oportunidade de mercado para a organização. Ou seja, o processo de design thinking gera ideias e, através de um processo criativo, propõe soluções que atendem às necessidades do mercado, com a tecnologia disponível no momento, combinada com o modelo de negócio da empresa.

Com base nos dados apresentados no quadro 1, e a partir da afirmação de Hobday, Boddington e Grantham (2012b), de que o design thinking propõe uma série de soluções para um dado problema, é possível afirmar que o método atende aos requisitos dos blocos de natureza e tipo.

\section{CONCLUSÕES}

O design thinking apresenta um fluxo de atividades que envolvem o processo de pesquisa (inspiração), geração de ideias e protótipos (ideação) e execução da solução (implementação), que contribuem para que o empresário compreenda quais são os recursos e habilidades necessárias para o desenvolvimento de uma inovação (Liedtka, 2011). Então, a fases do design thinking visam o desenvolvimento de uma solução para um problema específico, com foco no usuário a quem se destina tal solução. Tal definição vai ao encontro à descrição do processo de inovação de Verganti e Öberg (2013), que o caracterizam como um meio de resolução de problemas, de forma criativa, focada no ser humano.

A etapa de descoberta de oportunidade (imersão), visa a observação e pesquisa detalhada acerca do consumidor para compreender suas reais preocupações, aspirações e desejos, que serão transformados em ideias de soluções, que após testadas, serão ajustadas e inseridas no mercado. Para Sawhney, Wolcott e Arroniz (2011) a inovação só ganha relevância para uma empresa quando cria valor para os clientes e para a empresa. Ao entender o público-alvo e desenvolver o processo de ideação com agentes externos à empresa, a organização gera soluções mais condizentes com a demanda. Ao ofertar ao mercado produtos e serviços adequados às reais necessidades dos consumidores, a empresa alcança maior competitividade, ou seja, cria um valor para o cliente (produto) e para a organização (lucro).

A etapa de implementação deve ocorrer a partir da tecnologia praticável, da viabilidade para a empresa e da geração de valor para o consumidor, para então a solução definida ser planejada e produzida (Brown, 2008). Assim, o processo de design thinking vai ao encontro com o conceito de inovação de Barbieri (2004), que a define como a inserção de algo novo no mercado, observando-se os aspetos técnicos, mercadológicos, econômicos e organizacionais. Então, com base nas afirmações dos 
autores e na explanação na seção anterior, é possível afirmar que o design thinking gera inovação, e engloba todos os aspectos e características da inovação.

O design thinking também apresenta as características importantes dos modelos de inovação apresentadas na seção 2.2.2. A compreensão detalhada do comportamento do público-alvo contribui de sobremaneira no desenvolvimento de soluções que atendam às necessidades dos clientes. O design thinking incentiva o processo colaborativo, com ênfase para a interação entre todos da equipe (que pode englobar funcionários de diversos departamentos, fornecedores, clientes, consultores externos, etc.) e a difusão do conhecimento. A integração entre as oportunidades de mercado identificadas, as competências da empresa (tecnologia e pessoal) e o conhecimento dos diversos participantes do processo, aliado à criatividade, gera ideias diferentes e inovadoras.

O conceito de modelo de inovação engloba as atividades e todos os elementos necessários para desenvolver algo novo, tais como práticas, procedimentos, políticas e etc., compreendendo um mapa do processo de inovação (Longanezi, Coutinho \& Bomtempo, 2008; Oliveira, 2008). Cada uma das fases do design thinking se caracteriza por uma série de perguntas e atividades que orientam os participantes durante o processo para que se alcance os objetivos de cada etapa. Assim, devido às suas características e com base nas informações do referencial teórico, pode-se afirmar que o design thinking é um modelo de inovação.

Entretanto, os principal diferencial do design thinking em relação aos modelos de inovação mais recentes (a partir da década de 70), é o pensamento holístico, a partir de uma visão geral de equipe de inovação (sem delimitar departamentos e incluindo agentes externos) que utiliza a criatividade para co-criar novas ideias. O design thinking defende a geração de valor para os clientes (por meio de soluções mais adequadas às sua necessidades) e para empresa (através do alcance dos objetivos estratégicos).

Assim, o design thinking também apresenta os fatores importantes de um modelo de inovação, bem como os elementos essenciais para um processo de inovação, conforme critérios de Baregheh, Rowley e Sambrook (2009), sendo capaz de colaborar para as organizações gerarem inovações em diversas áreas (produto, serviço, processo, marketing e organizacional), englobando tanto inovações de caráter incremental, radical e semi-radical, contribuindo para as organizações alcancem seus objetivos estratégicos. Entretanto, o design thinking é mais adequado para processos de inovação aberta. Contudo, com base no que foi exposto, é plausível concluir que o design thinking pode ser caracterizado como um modelo de inovação que orienta o processo de geração de inovações. 


\section{REFERÊNCIAS}

Alvares, L. (2010). Gestão do conhecimento, aprendizado corporativo e inovação. Diretoria da Prodemge.

Barbieri, J. C. (2003). Organizações inovadoras: estudos e casos brasileiros. FGV Editora.

Baregheh, A., Rowley, J., \& Sambrook, S. (2009). Towards a multidisciplinary definition of innovation. Management decision, 47(8), 1323-1339.

Bonini, L. A., \& Sbragia, R. (2011). O modelo de design thinking como indutor da inovação nas empresas: um estudo empírico. Revista de Gestão e Projetos, 2(1).

Brown, T. (2008). Design thinking. Harvard business review, 86(6), 84.

Bukowitz, W. R. (2013). Fidelity Investments: adopting new models of innovation. Strategy \& Leadership, 41(2), 58-63.

Caraça, J., Lundvall, B. A., \& Mendonça, S. (2009). The changing role of science in the innovation process: From Queen to Cinderella? Technological Forecasting and Social Change, 76(6), 861-867.

Cardon, E. C., \& Leonard, S. (2010). Unleashing design: planning and the art of battle command. Army Combined Arms Center Fort Leavenworth Ks.

Chesbrough, H. (2012). Open innovation: where weve been and where were going. ResearchTechnology Management, 55(4), 20-27.

Cooper, R. G. (1990). Stage-gate systems: a new tool for managing new products. Business horizons, 33(3), 44-54.

Cooper, R. G. (1999). The invisible success factors in product innovation. Journal of product innovation management, 16(2), 115-133.

Cruz, T. (2003). Sistemas, métodos \& processos: administrando organizações por meio de processos de negócios.

Davila, T., Epstein, M. J., \& Shelton, R. (2008). As regras da inovação. Bookman.

Drucker, P. F. (2002). Administrando para obter resultados. Cengage Learning Editores.

Edquist, C., \& Hommen, L. (1999). Systems of innovation: theory and policy for the demand side. Technology in society, 21(1), 63-79.

Fraser, H. (2012). Design para negócios na prática: como gerar inovação e crescimento nas empresas aplicando o business design. Rio de Janeiro: Elsevier, 207.

Galvão, C. M., Sawada, N. O., \& Trevizan, M. A. (2004). Revisão Sistemática. Rev Latino-am enfermagem, 12(3), 549-56.

Godin, B. (2006). The Linear model of innovation the historical construction of an analytical 
framework. Science, Technology \& Human Values, 31(6), 639-667.

Gordon, S., Tarafdar, M., Cook, R., Maksimoski, R., \& Rogowitz, B. (2008). Improving the front end of innovation with information technology. Research-Technology Management, 51(3), 50-58.

Hacklin, F., Inganas, M., Marxt, C., \& Pluss, A. (2009). Core rigidities in the innovation process: a structured benchmark on knowledge management challenges. International Journal of Technology Management, 45(3), 244-266.

Hobday, M., Boddington, A., \& Grantham, A. (2012a). An Innovation Perspective on Design: Part 2. Design Issues, 28(1), 18-29.

Hobday, M., Boddington, A., \& Grantham, A. (2012b). Policies for design and policies for innovation: Contrasting perspectives and remaining challenges. Technovation, 32(5), 272-281.

Hronszky, I., \& Kovács, K. (2013). Interactive Value Production through Living Labs. Acta Polytechnica Hungarica, 10(2).

İzadi, A., Zarrabi, F., \& Zarrabi, F. (2013). Firm-level innovation models. Procedia-Social and Behavioral Sciences, 75, 146-153.

Kline, S. J., \& Rosenberg, N. (1986). An overview of innovation. The positive sum strategy: Harnessing technology for economic growth, 275, 305.

Koen, P., Ajamian, G., Burkart, R., Clamen, A., Davidson, J., D'Amore, R., lkins, C., Herald, K., Incorvia, M., Johnson, A., Karol, R., Seibert, R., Slavejkov, A. \& Wagner, K. (2001). Providing clarity and a common language to the" fuzzy front end". Research-Technology Management, 44(2), 46-55.

Liedtka, J. (2011). Learning to use design thinking tools for successful innovation. Strategy \& Leadership, 39(5), 13-19.

Liem, A., \& Brangier, E. (2012). Innovation and design approaches within prospective ergonomics. Work: A Journal of Prevention, Assessment and Rehabilitation, 41, 5243-5250.

Longanezi, T., Coutinho, P., \& Bomtempo, J. V. M. (2008). Um modelo referencial para a prática da inovação. Journal of Technology Management \& Innovation, 3(1).

Miguel, P. A. C. et al. (2009). Desafios para a metodologia de pesquisa em Engenharia de Produção. In Oliveira, V. F., Cavenaghi, V., \& Másculo, F. S. [Orgs]. Tópicos emergentes e desafios metodológicos em Engenharia de Produção: casos, experiências e proposições. Rio de Janeiro: ABEPRO.

Mlecnik, E. (2013). Opportunities for supplier-led systemic innovation in highly energy-efficient housing. Journal of Cleaner Production, 56, 103-111.

Monteiro, E. V. (2012). Perfis de liderança. Retirado de http://economico.sapo.pt/noticias/perfis-delideranca_149445.html

Oliveira, S. B. D. (2008). Gestão por processos: fundamentos, técnicas e modelos de implementação: foco no sistema de gestão da qualidade com base na ISO 9000: 2005 e ISO 9001. Rio de Janeiro: Qualitymark. 
Organização para Cooperação e Desenvolvimento Econômico. (2004). Manual de Oslo: Proposta de diretrizes para coleta e interpretação de dados sobre inovação tecnológica. Brasília: OCDE, FINEP.

Osterwalder, A.; Pigneur, Y. (2011). Business Model Generation - inovação em modelos de negócios: um manual para visionários, inovadores e revolucionários. Alta Books, p.300.

Pärttö, M., \& Saariluoma, P. (2012). Explaining failures in innovative thought processes in engineering design. Procedia-Social and Behavioral Sciences, 41, 442-449.

Rodrigues, L. C., de França Heringer, B. H., \& França, A. L. (2010). Padrões de inovação em multinacional de base tecnológica. Revista de Administração e Inovação-RAI, 7(3).

Rothwell, R. (1994). Towards the fifth-generation innovation process. International marketing review, 11(1), 7-31.

Saraiva, M., \& Rolo, A. (2009). Qualidade e inovação: principais implicações nas organizações portuguesas.

Sawhney, M., Wolcott, R. C., \& Arroniz, I. (2011). The 12 different ways for companies to innovate. Top 10 Lessons on the New Business of Innovation, 47, 28.

Schumpeter, J. A., \& García, J. D. (1983). Capitalismo, socialismo y democracia.

Seuring, S., \& Gold, S. (2012). Conducting content-analysis based literature reviews in supply chain management. Supply Chain Management: An International Journal, 17(5), 544-555.

Teece, D. J. (1983). Business Models, Business Strategy and Innovation. Long Range Planning, v. 43, n. 2-3, p. 172-194.

Tidd, J., Bessant, J., \& Pavitt, K. (2008). Gestão da inovação. Bookman.

Tohidi, H., \& Jabbari, M. M. (2012). Different stages of innovation process. Procedia Technology, 1, 574-578.

Verganti, R., \& Öberg, Å. (2013). Interpreting and envisioning-A hermeneutic framework to look at radical innovation of meanings. Industrial Marketing Management, 42(1), 86-95.

Wang, J., \& Kleiner, B. H. (2005). The evolution of R\&D management. Management research news, 28(11/12), 88-95.

Wylant, B. (2008). Design thinking and the experience of innovation. Design Issues, 24(2), 3-14. 


\title{
THE CHARACTERISTICS OF THINKING AS A DESIGN INNOVATION MODEL
}

\begin{abstract}
To organizations stay competitive, it's important offer innovative solutions to the market, meeting the consumer's needs. To create innovations aligned with the business strategies, processes are required to coordinate the activities of different departments of the company, in other words, is necessary to develop an innovation process in the organization. Over last decade the design thinking has been used to promote innovation in the organizations, so, the question is if the design thinking can help to create innovation in the organizations, and if it can generate all the types of innovation. Thus, the objective of this study is to characterize design thinking as a model of innovation. The research is theoretical and conceptual, through a review of the literature of innovation and analysis of publications with empirical evidence of design thinking. The analysis of empirical data showed that design thinking is indicated to open innovation, and is able to generate radical innovations, semi radical innovations and incremental innovations, and can be used to develop different types of innovation (product, service, process, organizational and marketing). Based in the essential requirements of an innovation process and the main features of a model of innovation, the research concluded that the design thinking could be characterized as a model of innovation.
\end{abstract}

Key words: Innovations; Innovation processes; Innovation models; Design thinking.

Data do recebimento do artigo: 21/07/2014

Data do aceite de publicação: 07/05/2015

Revista de Administração e Inovação, São Paulo, v. 12, n.3 p. 157-182, jul./set. 2015. 